\title{
Supplementation of Superfine Powder Prepared from Chaenomeles speciosa Fruit Increases Endurance Capacity in Rats via Antioxidant and Nrf2/ARE Signaling Pathway
}

\author{
Ka Chen, Jia You, Yong Tang, Yong Zhou, Peng Liu, Dan Zou, Qicheng Zhou, \\ Ting Zhang, Jundong Zhu, and Mantian Mi
}

Research Center for Nutrition and Food Safety, Institute of Military Preventive Medicine, Third Military Medical University, Shapingba District, Chongqing 400038, China

Correspondence should be addressed to Mantian Mi; mimantian@sina.com

Received 29 August 2014; Accepted 17 November 2014; Published 24 December 2014

Academic Editor: Jairo K. Bastos

Copyright (C) $2014 \mathrm{Ka}$ Chen et al. This is an open access article distributed under the Creative Commons Attribution License, which permits unrestricted use, distribution, and reproduction in any medium, provided the original work is properly cited.

\begin{abstract}
Chaenomeles speciosa fruit is a traditional herb medicine widely used in China. In this study, superfine powder of C. speciosa fruit (SCE), ground by supersonic nitrogen airflow at $-140^{\circ} \mathrm{C}$, was investigated to assess its in vitro antioxidant activity and in vivo antiphysical fatigue activity. SCE was homogenous $(d<10 \mu \mathrm{m})$ and rich in antioxidants like polyphenols, saponins, oleanolic acid, ursolic acid, ascorbic acid, and SOD. According to the in vitro experiments, SCE displayed promising antioxidant activity with powerful FARP, SC-DPPH, and SC-SAR activities. According to the in vivo experiments, rats supplemented with SCE had prolonged exhaustive swimming time (57\%) compared to the nonsupplemented rats. Meanwhile, compared to the nonsupplemented rats, the SCE-supplemented rats had higher levels of blood glucose and liver and muscular glycogen and lower levels of LA and BUN. Lower MDA, higher antioxidant enzymes (SOD, CAT, and GSH-Px) activities, and upregulated Nrf2/ARE mediated antioxidant enzymes (HO-1, Trx, GCLM, and GCLC) expression were also detected in the supplemented group. This study indicates that SCE is a potent antioxidant and antifatigue agent, and SCE could be a promising raw material for the food and pharmaceutical industries.
\end{abstract}

\section{Introduction}

Exercise-induced fatigue has been extensively studied in athletes, military, and industry personnel. Exercise intolerance, characterized by difficulty in sustaining voluntary activities, is the well-known consequence of this physical fatigue. Although the underlying mechanism of exercise-induced fatigue has not been fully clarified, reactive oxygen species (ROS), especially those derived from skeletal muscle, have been regarded as the crucial factor in fatigue development and progress [1]. In recent studies, several natural antioxidants, such as green tea extract [2,3], ginsengs [4], red mold rice [5], and maca extract [6], have been proved to be effective in improving physical endurance. So, people pay more attention to seek natural compounds with potent antioxidant activity to improve physical performance, postpone fatigue, and accelerate the elimination of fatigue in human beings.
Chaenomeles speciosa (Sweet) Nakai (Rosaceae) is a traditional Chinese herb widely used in the treatment of dyspepsia and several inflammatory diseases. Recent studies have reported its immunomodulatory effect, hepatoprotective effect, antinociceptive effect, antitumor effect, and antihyperlipidemic effect [7-10]. As a result of its various health effects revealed, C. speciosa has gained considerable attention both in Chinese medicine and in food industry. Studies have reported that polyphenols (e.g., phenolics, flavonoids, quercetin, etc.), alkaloids, organic acids, glycosides, and some amino acid derivatives are the main bioactive components of $C$. speciosa fruits [7-10]. Among these ingredients, polyphenols, saponins, oleanolic acid (OA), ursolic acid (UA), ascorbic acid, and superoxide dismutase (SOD), which are powerful antioxidants [7, 10], might have antifatigue properties. However, there are no published reports on the antifatigue property of C. speciosa produced in China. 
In the present study, we identified whether SCE could produce positive effects on endurance capacity. To maintain the important bioactive components and avoid the incorporation of additional chemical agents, a modified superfine grinding process, which included flash evaporation and superfine grinding using a 3.08 Mach supersonic airflow at $-140^{\circ} \mathrm{C}$, was used in the preparation of SCE. In this work, quality characters of SCE such as particle size, the main antioxidants contents, and in vitro antioxidant activities were firstly analyzed. Then, the in vivo antifatigue activity was assessed using the weighted forced swim test (WFST) with rats feeding with SCE for 14 days, and biomarkers related to fatigue and oxidative stress were measured, and the nuclear factor erythroid 2-related factor 2 (Nrf2)/antioxidant response element (ARE) signaling pathway in skeletal muscle was further detected. This work would provide an important basis for developing SCE as a novel antifatigue agent and facilitate further development and utilization of C. speciosa.

\section{Materials and Methods}

2.1. Chemicals. Gallic acid, ellagic acid, chlorogenic acid, caffeic acid, catechin, quercetin, thiobarbituric acid, DPPH, 2, 4, 6-tripyridyl-s-triazine (TPTZ), and ferrozine were purchased from Sigma Chemical Co. (St. Louis, MO, USA). OA and UA standard were obtained from Siyi Biotechnology Company (Chengdu, China). Reagents for measuring SC-SAR, TAOC, thiobarbituric acid reacting substances (TBARS), total GSH, SOD, CAT, GSH-Px, and BUN were bought from Nanjing Jiancheng Institute of Biology and Engineering (Nanjing, China). cDNA synthesis kit and real-time PCR were purchased from Bio-Rad (Hercules, CA). Primers for HO-1, Trx, GCLM, GCLC, and $\beta$-actin were purchased from Invitrogen (Shanghai, China). Primary antibody against Nrf2 and Lamin $B$ were obtained from Abcam (Cambridge, MA) and other antibodies for detection of HO-1, Trx, GCLC, GCLM, and $\beta$-actin were from Novus Biologics (Littleton, CO). All the organic solvents and chemicals used in this study were of analytical grade.

2.2. SCE Preparation. C. speciosa fruit was collected from the Qijiang district of the city of Chongqing in China during the early month of September (2012) and was stored at $-20^{\circ} \mathrm{C}$ until used. The C. speciosa fruit was washed, enucleated, and ground into coarse powder $(d<30$ mesh). Then, the coarse powder was subjected to an automatic flash dryer. When the water content of coarse powder was $<8 \%$, the powder was then ground to achieve a superfine powder consistent in an UF-250 airflow micronizer (Saishan Powder Machinery Manufacturing Co. Ltd., Shanghai, China) with a 3.08 Mach supersonic nitrogen airflow at $-140^{\circ} \mathrm{C}$. Meanwhile, the traditional herbal pieces for decoction of C. speciosa fruit (TCE) were processed according to the Chinese pharmacopoeia (2010 version). TCE, which served as the control, was analyzed for appraising the antioxidant quality indexes of SCE.

2.3. Morphological Character and Particle Size Distribution Analysis of SCE. Morphological characterization of SCE particles was performed on images acquired using a scanning electron microscope (SEM). The particle size distribution of SCE was measured by a laser diffraction instrument (Mastersizer 2000, UK).

2.4. The Main Antioxidants Contents of SCE Analysis. HPLC analysis of OA, UA, and the representative phenolics including gallic acid, ellagic acid, chlorogenic acid, caffeic acid, catechin, and quercetin was carried out on an Alliance HPLC system (Waters, USA; see Materials and Methods in Supplementary Material available online at http://dx.doi.org/10.1155/2014/976438). TPC was measured by Folin-Ciocalteu assay and total saponins were detected using spectrophotometry method as previously described [7]. Ascorbic acid detection was followed by 2,6,-dichlorophenol-indophenol titrimetric analysis and the activity of SOD detection used the total SOD assay kit with WST-1 [7].

2.5. Assessment of the In Vitro Antioxidant Activity of SCE. The SC-DPPH, FRAP assays were measured according to the methods reported by us [7]. And the SC-SAR assay was detected using a commercial kit.

\subsection{Assessment of the In Vivo SCE Antioxidant and Antifatigue Activities}

2.6.1. Animals and Experimental Design. Forty adult SpragueDawley rats (weight: $210 \pm 10 \mathrm{~g}$ ) were obtained from the Experimental Animal Center of the Third Military Medical University. After one week on an AIN-93 diet, rats were randomly divided into two groups: control group (Con.) and SCE group (SCE). The SCE group and Con. group, respectively, received $1.0 \mathrm{~g} / \mathrm{kg}$ body weight of SCE in $10 \%$ PBS solution (intragastric gavage (ig)) or 10\% PBS for 17 days at 9:00 am. From the 1st to the 13th day, each group underwent a swimming test for 5 min without any weights; the swimming test was performed between 1:00 and 3:00 pm. On the 14th day, each group underwent a WFST. According to the results obtained from the WFST, the rats were further divided into four groups: nonexercise (NEx) or control group, nonexercise with SCE supplementation group (NEx + SCE), exercise group (Ex), and exercise with SCE supplementation group $(\mathrm{Ex}+\mathrm{SCE})$. On the last three days, the Ex and Ex + SCE groups underwent an exhaustive exercise every day (i.e., WFST for $20 \mathrm{~min}$, twice each day) to induce physical fatigue. The other two groups were allowed to rest. Following the 18th day, the animals were sacrificed and blood samples, liver, and gastrocnemius muscle were quickly obtained. All animal procedures were performed according to the Third Military Medical University Institutional Animal Care and Use Committee approved protocols.

2.6.2. WFST. During 7 days, rats underwent a swimming test for 5 min without any weights. The WFST was subsequently performed in a round tank $(100 \times 50 \times 50 \mathrm{~cm})$ maintained at $33^{\circ} \mathrm{C}$ with a $75 \mathrm{~cm}$ water depth. The rats performed the WFST until they reached exhaustion. The weight, which was attached to their tails, was approximately $5 \%$ of their body 


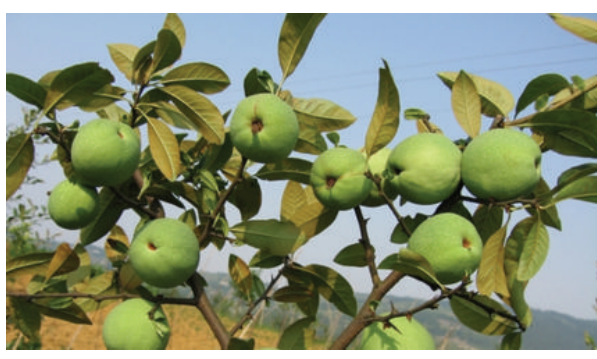

(a)

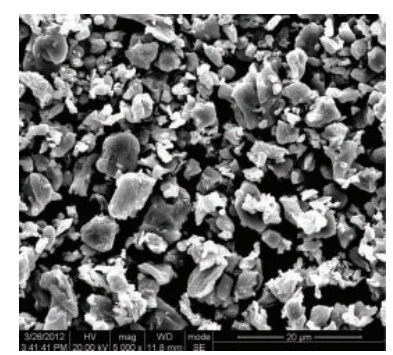

(b)

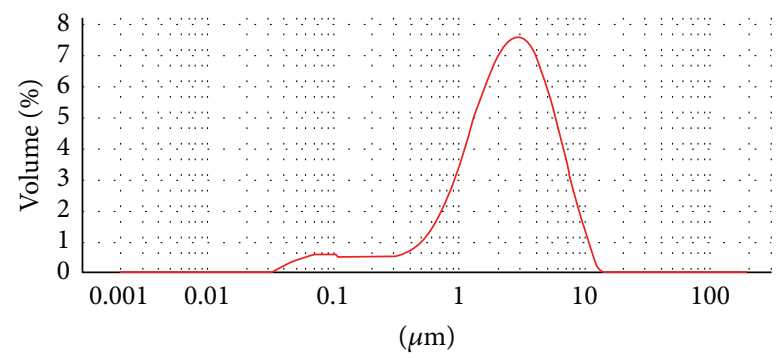

(c)

FIGURE 1: SEM images of the superfine powder from C. speciosa fruit (SCE) and its particles distribution diagram. (a) Fresh fruit of C. speciosa; (b) representative SEM images of SCE; (c) particles distribution diagram of SCE.

TABle 1: Primers for real-time PCR assay.

\begin{tabular}{ll}
\hline Gene & Primer $\left(5^{\prime}-3^{\prime}\right)$ \\
\hline \multirow{2}{*}{ HO-1 } & F: ATGACACCAAGGACCAGAGC \\
& R: GTAAGGACCCATCGGAGAAGC \\
\hline \multirow{2}{*}{ Trx } & F: CTGCTTTTCAGGAAGCCTTG \\
& R: TGTTGGCATGCATTTGACTT \\
\multirow{2}{*}{ GCLM } & F: ACTGACTTAGGAGCATAACTTACC \\
& R: AAGAATATCTGCCTCAATGACACC \\
\hline \multirow{2}{*}{ GCLC } & F: AAGCCATTCACTCCAGATTTTACC \\
& R: ACAACAAACTTCAACGCAAAGC \\
\hline \multirow{2}{*}{$\beta$-actin } & F: GCCGCCAG CTCACCATGGATG \\
& R: GACCCCGTCACCGGAGTC CA \\
\hline
\end{tabular}

weights. It was assumed that the rats had reached exhaustion when they failed to rise to the surface to breathe within a period of $10 \mathrm{~s}$.

2.6.3. Measuring Biochemical Parameters Related to Fatigue and Redox System. The levels of glucose, glycogen, LA, BUN, MDA, SOD, TAOC, CAT, and GSH-Px were measured using commercial kits.

2.6.4. Real-Time PCR and Western Blot Analyses. Total RNA $(1 \mu \mathrm{g})$ from the gastrocnemius muscle was used to perform real-time PCR with the SYBR Green PCR Master Mix kit. Primers for HO-1, Trx, GCLM, GCLC, and $\beta$-actin were purchased from Invitrogen (Shanghai, China; see Table 1). The nuclear or total protein of gastrocnemius muscle was blotted with the primary antibodies against Nrf2, Lamin B, GSH, Trx, GCLC, GCLM, and $\beta$-actin. The band optical density was determined by Quantity One software (Bio-Rad Lab, Hercules, CA, USA).

2.7. Statistical Analyses. The experimental data are expressed as mean \pm standard error and analyzed with the SPSS 17.0 software. The exhaustive swimming time data were analyzed by an unpaired sample $t$-test. The other results were analyzed by two-way ANOVA and Tukey's post hoc test. The significance level was set at $P<0.05$.

\section{Results and Discussion}

3.1. Powder Yield and Particle Size of the Prepared SCE. C. speciosa is a traditional herb that has been cultivated in China for thousands of years (Figure 1(a)). The traditional use of C. speciosa fruit, such as the TCE, cannot fulfill the variety demand. Although several technologies including extractions with enzymes and ethanol have been used in the deep processing of C. speciosa, bioactive components loss and chemical agents incorporation were big problems that we were confronted with $[7,10]$. Nowadays, superfine grinding technology has begun to be used in foods processing, which would lead to better dispersibility, higher nutrient solubility, and bioavailability [11-14]. In this study, a modified superfine grinding technology was used to prepare SCE. Fresh fruit $(14.0 \mathrm{~kg})$ was processed to $1.82 \mathrm{~kg}$ powder and the yield of SCE was about 13\%. During the SCE preparation, no chemicals were additionally added thereby ensuring its safety and purity. The morphology after milling changed considerably (Figure 1(b)), and the particle size of SCE was mainly distributed in 1-10 $\mu \mathrm{m}$ (Figure $1(\mathrm{c})$ ). A particle size of $0.5-10 \mu \mathrm{m}$ (in diameter) can significantly increase nutrient leaching, and superfine powder is easily incorporated into 
TABLE 2: The main antioxidants contents and in vitro antioxidant activity of SCE.

\begin{tabular}{lcc}
\hline & SCE & TCE \\
\hline Oleanolic acid (\%) & $0.326 \pm 0.064^{*}$ & $0.208 \pm 0.031$ \\
Ursolic acid (\%) & $0.189 \pm 0.031^{*}$ & $0.143 \pm 0.015$ \\
Total flavones (mg/g) & $49.15 \pm 4.18^{*}$ & $25.24 \pm 2.63$ \\
$\quad$ Gallic acid (mg/g) & $6.80 \pm 0.32^{*}$ & $4.38 \pm 0.22$ \\
$\quad$ Ellagic acid (mg/g) & $9.42 \pm 1.45^{*}$ & $6.42 \pm 1.73$ \\
$\quad$ Chlorogenic acid (mg/g) & $2.01 \pm 0.38^{*}$ & $1.24 \pm 0.26$ \\
$\quad$ Caffeic acid (mg/g) & $1.57 \pm 0.31^{*}$ & $0.34 \pm 0.26$ \\
$\quad$ Catechin (mg/g) & $1.95 \pm 0.65^{*}$ & $0.75 \pm 0.23$ \\
$\quad$ Quercetin (mg/g) & $11.28 \pm 2.39^{*}$ & $7.03 \pm 1.51$ \\
Total saponins (mg/g) & $18.105 \pm 0.623^{*}$ & $11.214 \pm 0.409$ \\
Ascorbic acid (mg/g) & $3.52 \pm 0.83^{*}$ & $0.09 \pm 0.02$ \\
Superoxide dismutase activity & $68.895 \pm 5.35^{*}$ & $3.125 \pm 1.23$ \\
(U/mg) $_{\text {SCD }}$ (mg DPPH/g) & $4.15 \pm 1.81^{*}$ & $1.28 \pm 0.6$ \\
SC-SAR $^{\mathrm{b}}(\mathrm{U} / \mathrm{g})$ & $2207 \pm 163^{*}$ & $848 \pm 94$ \\
FRAP $^{\mathrm{c}}$ (mmol Fe & & \\
\hline
\end{tabular}

Values represent means $\pm \mathrm{SD} ; n=3 ;^{*} P<0.05$ versus TCE group; ${ }^{\mathrm{a}} \mathrm{SC}-$ $\mathrm{DPPH}$, scavenging capacity of DPPH.(1,1-diphenyl-2-picrylhydrazyl free radical); ${ }^{\mathrm{b}}$ SC-SAR, scavenging capacity of superoxide anion radical; ${ }^{\mathrm{c}}$ FRAP, ferric reducing antioxidant power.

food matrices $[13,14]$. Therefore, SCE may contribute to higher nutrient absorption and is suitable in the development of convenient food and pharmaceutical products.

3.2. Antioxidants Contents of the Prepared SCE. Previous studies have reported that the presence of oxygen and heat increases polyphenol and other antioxidants degradation. Some studies have reported that polyphenols such as EGCG, EGC, and EC are considerably reduced during superfine grinding [14]. In this study, to reduce the ruin effects of oxygen and heat on antioxidants, a coarse powder of $C$. speciosa fruit underwent superfine grinding by a $3.08 \mathrm{Mach}$ supersonic nitrogen airflow at $<-140^{\circ} \mathrm{C}$; in these conditions, oxygen exposure and high temperatures were prevented. Phytochemical investigations on C. speciosa fruit reported that many classes of chemical groups contributed to its varieties of pharmacological function [7-10]. As shown in Table 2, superfine grinding significantly increased OA and UA, total polyphenols, gallic acid, ellagic acid, chlorogenic acid, caffeic acid, catechin, and quercetin concentrations when compared to TCE. To further determine the in vitro antioxidant activity of SCE, radical scavenging and reducing activity tests were performed. As shown in Table 2, SCE had higher DPPH $\mathrm{IC}_{50}$ value, SC-SAR activity, and FRAP activity than that of TCE. Above all, the development of the modified superfine grinding yielded a final product which is homogeneous and rich in antioxidants with powerful antioxidant activities.

3.3. SCE Prolonged the Exhaustive Swimming Time. In our preliminary experiments, a range of doses $(0.2,1.0$, and $5.0 \mathrm{~g} / \mathrm{kg}$ body weight/day) of SCE were studied to determine the most effective dose. Unfortunately, during the supplementation period, the $5.0 \mathrm{~g} / \mathrm{kg}$ SCE dose resulted in loss of appetite and a reduction in body weight. Studies have suggested that polyphenols have strong effects on body tissues (e.g., severe chemical burns); thus the above symptoms might have been attributed to the high polyphenol content of SCE [7]. With the other two SCE doses, however, there were no obvious side effects. Based on the preliminary experiments (data not shown), the chosen SCE dose was $1.0 \mathrm{~g} / \mathrm{kg}$ body weight/day.

A direct measure of antifatigue effect is the increase in exercise tolerance. WFST is a classical exercise model to evaluate antifatigue; it works well for evaluating the endurance of mice/rats and is highly reproducible [15-18]. Reduced susceptibility to fatigue is correlated with longer exhaustive swimming times. In this study, SCE significantly prolonged exhaustive swimming time from approximately $7 \mathrm{~min}$ to $11 \mathrm{~min}$ (Figure 2(a)). The results indicate that SCE has antifatigue activity and could increase endurance.

3.4. Effects of SCE on Biomarkers Related to Fatigue. Blood glucose homeostasis plays an important role in increasing endurance. Glycogen, an important energy source during exercise, maintains blood glucose within a physiological range. The amount of glycogen stored in the body reflects the speed and degree to which fatigue will occur [15-18]. As shown in Figure 2(b), SCE inhibited the exhaustive exerciseinduced reduction of the blood glucose and the muscle and liver glycogen levels $(P<0.05)$. Next, we detected the sensitive fatigue indicators including LA and BUN, which were metabolism products of carbohydrate and protein, respectively [15-18]. Results showed that SCE reduced the exhaustive exercise-induced elevation of serum LA and BUN (Figure $2(\mathrm{c}), P<0.05)$. These results suggest that the blood glucose-homeostatic ability and reduced accumulated byproducts of metabolism may be related to an improvement in exercise metabolism and antifatigue activity of SCE.

\subsection{SCE Enhanced Antioxidative Enzymes in Rats That Under-} went Exhaustive Exercise. In this study, we measured serum markers of oxidative stress including MDA, TAOC, SOD, GSH-Px, and CAT. MDA is one of the end-products of lipid peroxidation process; its levels increase during strenuous exercises $[10,19]$. As shown in Figure 2(d), after three days of exhaustive exercise, the MDA level of the Ex + SCE group was lower than that of the Ex group $(P<0.05)$. TAOC was measured to assess the serum total antioxidant status. Serum antioxidant enzymes including SOD, GSH-Px, and CAT are important for scavenging free radicals and for maintaining normal cellular physiology $[15,20,21]$. In the present study, SCE inhibited the exhaustive exercise reduction of serum TAOC and activities of SOD, GSH-Px, and CAT (Figure 2(d)). These results provide evidence that SCE supplementation attenuates the exercise-induced oxidative stress and helps restore the oxidant-antioxidant balance.

3.6. SCE Regulated Nrf2/ARE Pathway in Rats That Underwent Exhaustive Exercise. Nrf2 was reported to play a key role in 


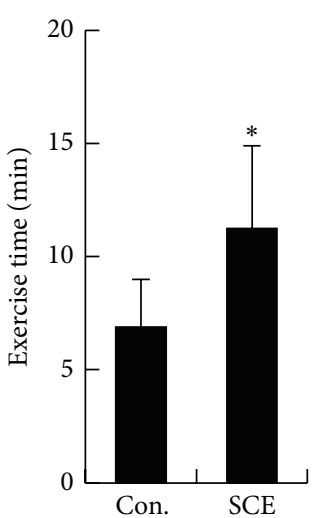

(a)

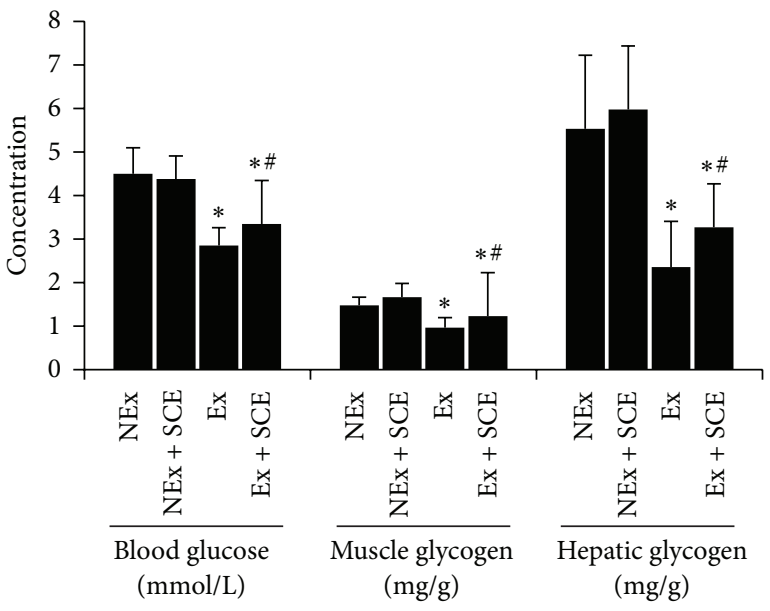

(c)

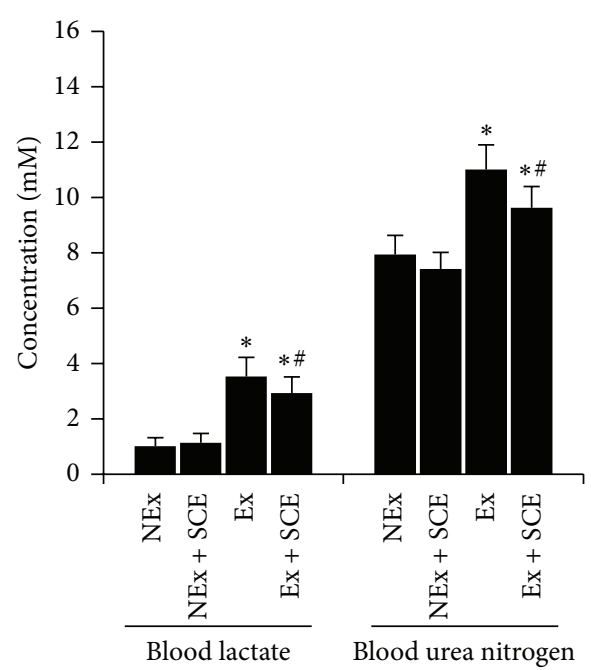

(b)

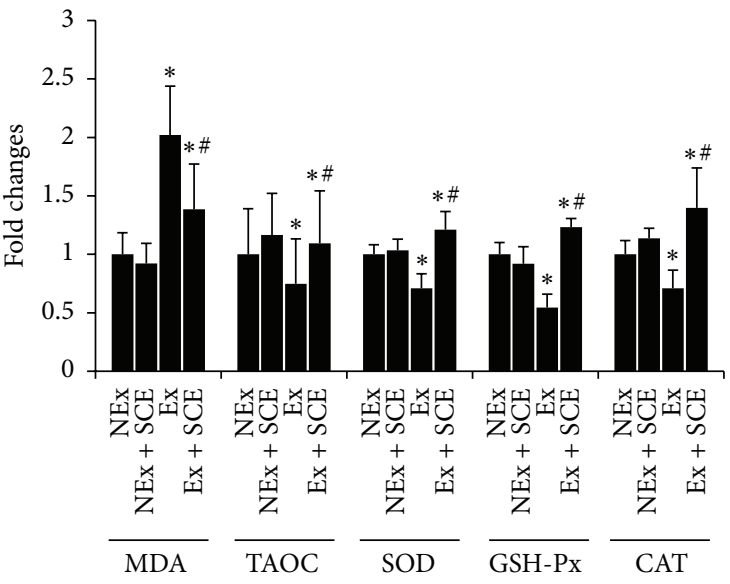

(d)

FIGURE 2: Antifatigue effect of the superfine powder prepared from C. speciosa fruit (SCE). (a) Exhaustive swimming time of rats in WFST test. Values represent mean \pm SE. ${ }^{*} P<0.05$ versus Con.: control group; SCE: SCE-supplemented group. (b) Blood lactic acid and blood urea nitrogen of rats exposed to exhaustive exercise. (c) Blood glucose, muscle glycogen, and liver glycogen of rats exposed to exhaustive exercise. (d) Oxidative-antioxidative statue of rats exposed to exhaustive exercise. Fold change was equal to that of the corresponding NEx group. Values represent mean $\pm \mathrm{SE} .{ }^{*} P<0.05$ versus NEx group; ${ }^{\#} P<0.05$ versus Ex group. NEx: nonexercise group; NEx + SCE: nonexercise with SCE supplementation group; Ex: exercise group; Ex + SCE: exercise with SCE supplementation group.

regulating oxidative stress [22, 23]. Under basal conditions, Nrf2 is sequestered in the cytoplasm by Keap1 and rapidly degraded in an ubiquitin-proteasome-dependent manner, whereas under conditions of oxidative stress, Nrf2 escapes Keap1-mediated repression and translocates into nucleus to activate the expression of antioxidant and phase II drugmetabolizing enzymes through ARE. Recent studies have revealed that Nrf2-dependent modulation of redox system was the alternative mechanisms of flavones (quercetin and curcumin) cell protection, which is beyond its longestablished ROS scavenging properties [22, 23]. In this study, although increased nuclear location of Nrf2 was observed (Figure 3(a)), the MDA accumulation displayed exhaustive exercise-induced oxidative stress in rats of Ex group. However, SCE enhanced the fatigue-induced upregulation of Nrf2 and expression of HO-1, Trx, GCLC, and GCLM, which were downstream antioxidative genes product of the Nrf2/ARE pathway (Figures 3(b) and 3(c)). Thus, modulation of Nrf2/ARE signal pathway is likely to play a critical role in prevention of the muscle fatigue from the oxidative damage by SCE.

\section{Conclusions}

This study showed that SCE prepared by a superfine grinding technology has not only in vitro antioxidant activities but also in vivo antifatigue effects in rats. SCE has an ultrafine particle size and high content of antioxidants. The in vivo experiments study revealed that rats supplemented with SCE exhibited an increase in exhaustive swimming time. Meanwhile, improved exercise metabolism and activated energy metabolic reactions as evidenced by the increasing levels of 


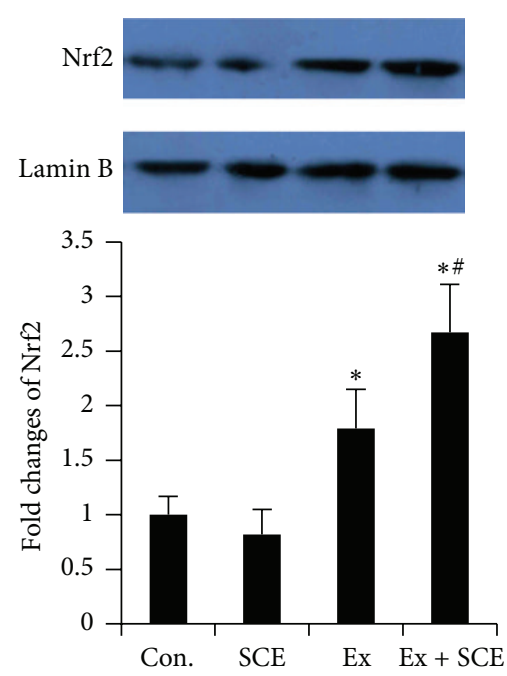

(a)

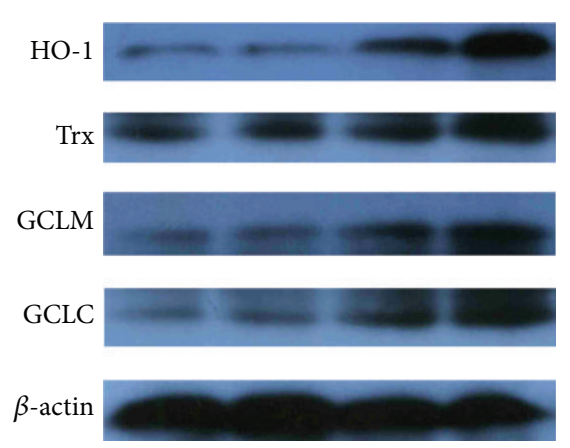

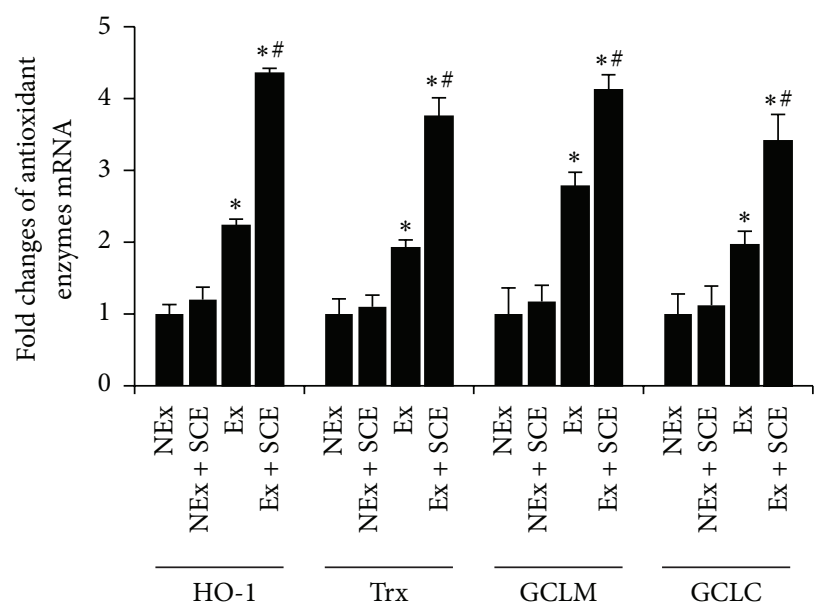

(b)

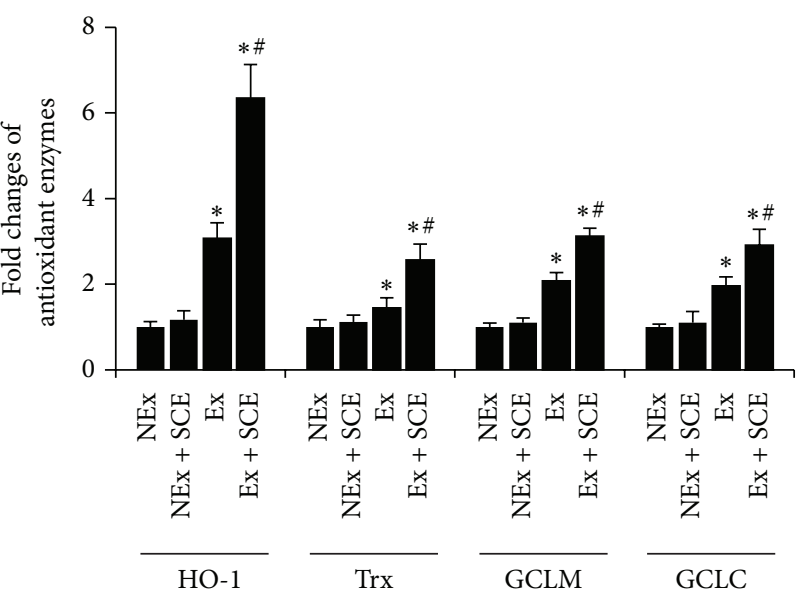

(c)

Figure 3: Effect of the superfine powder prepared from C. speciosa fruit (SCE) on Nrf2/ARE signal pathway of rats exposed to exhaustive exercise. (a) Nrf2 protein levels in gastrocnemius muscle. (b) mRNA levels of ARE related antioxidant enzymes in gastrocnemius muscle. (c) Protein levels of ARE related antioxidant enzymes in gastrocnemius muscle. Fold changes were quantified as the target protein or mRNA levels equal to the corresponding internal control (Lamin B or $\beta$-actin) in the NEx group. Values represent mean $\pm \mathrm{SE}$. ${ }^{*} P<0.05$ versus NEx group; ${ }^{\#} P<0.05$ versus Ex group. NEx: nonexercise group; NEx + SCE: nonexercise with SCE supplementation group; Ex: exercise group; Ex + SCE: exercise with SCE supplementation group.

blood glucose and of liver and muscle glycogen were observed in rats supplemented with SCE. Furthermore, rats supplemented with SCE showed a reduction of the accumulated by-products of metabolism, an inhibition of exercise-induced lipid peroxidation, and an improvement of the endogenous cellular antioxidant capacity by increasing the activities of antioxidant enzymes and upregulating Nrf2/ARE mediated antioxidant enzymes expression. Therefore, SCE supplementation can increase endurance capacity and facilitate recovery from fatigue in rats. The results provide an important basis for developing SCE as a novel antioxidant and antifatigue compound.

\section{Abbreviations}

ARE: Antioxidant response element

BUN: Blood urea nitrogen
CAT: $\quad$ Catalase

GSH-Px: Glutathione peroxidase

LA: $\quad$ Lactic acid

TAOC: Total antioxidative capacity

TPC: $\quad$ Total flavones content

MDA: Malondialdehyde

SC-DPPH: 1,1-Diphenyl-2-picrylhydrazyl scavenging capacity

FARP: $\quad$ Ferric reducing antioxidant power

PBS: $\quad$ Phosphate buffer solution

SC-SAR: Superoxide anion radical scavenging capacity

SOD: $\quad$ Superoxide dismutase

Nrf2: $\quad$ Nuclear factor erythroid 2-related factor 2

HO-1: Heme oxygenase-1

Trx: Thioredoxins

GCLC: Glutamate-cysteine ligase catalytic subunit

GCLM: Glutamate-cysteine ligase modifier subunit. 


\section{Conflict of Interests}

All authors confirmed that they have no conflict of interests.

\section{Acknowledgment}

This work was funded by research grants from the key projects of the "Twelfth Five-Year Plan" for Medical Science Development of PLA, China (BWS12J034).

\section{References}

[1] M. B. Reid, "Free radicals and muscle fatigue: of ROS, canaries, and the IOC," Free Radical Biology and Medicine, vol. 44, no. 2, pp. 169-179, 2008.

[2] A. Singal, S. Kaur, N. Tirkey, and K. Chopra, "Green tea extract and catechin ameliorate chronic fatigue-induced oxidative stress in mice," Journal of Medicinal Food, vol. 8, no. 1, pp. 47-52, 2005.

[3] M. Tanaka, Y. Baba, Y. Kataoka et al., "Effects of (-)-epigallocatechin gallate in liver of an animal model of combined (physical and mental) fatigue," Nutrition, vol. 24, no. 6, pp. 599603, 2008.

[4] M. Etemadifar, F. Sayahi, S. H. Abtahi et al., "Ginseng in the treatment of fatigue in multiple sclerosis: a randomized, placebo-controlled, double-blind pilot study," International Journal of Neuroscience, vol. 123, pp. 480-486, 2013.

[5] J.-J. Wang, M.-J. Shieh, S.-L. Kuo, C.-L. Lee, and T.-M. Pan, "Effect of red mold rice on antifatigue and exercise-related changes in lipid peroxidation in endurance exercise," Applied Microbiology and Biotechnology, vol. 70, no. 2, pp. 247-253, 2006.

[6] E. H. Choi, J. I. Kang, J. Y. Cho et al., "Supplementation of standardized lipid-soluble extract from maca (Lepidium meyenii) increases swimming endurance capacity in rats," Journal of Functional Foods, vol. 4, no. 2, pp. 568-573, 2012.

[7] Y. Tang, X. Yu, M. Mi, J. Zhao, J. Wang, and T. Zhang, "Antioxidative property and antiatherosclerotic effects of the powder processed from chaenomeles speciosa in apoe ${ }^{-/-}$mice," Journal of Food Biochemistry, vol. 34, no. 3, pp. 535-548, 2010.

[8] Q. Zhu, C. Liao, Y. Liu et al., "Ethanolic extract and watersoluble polysaccharide from Chaenomeles speciosa fruit modulate lipopolysaccharide-induced nitric oxide production in RAW264.7 macrophage cells," Journal of Ethnopharmacology, vol. 144, no. 2, pp. 441-447, 2012.

[9] Q. Zhu, C. Liao, Y. Liu et al., "Ethanolic extract and watersoluble polysaccharide from Chaenomeles speciosa fruit modulate lipopolysaccharide-induced nitric oxide production in RAW264.7 macrophage cells," Journal of Ethnopharmacology, vol. 144, no. 2, pp. 441-447, 2012.

[10] S. Y. Zhang, L. Y. Han, H. Zhang, and H. L. Xin, "Chaenomeles speciosa: a review of chemistry and pharmacology," Bioscience Reports, vol. 2, no. 1, pp. 12-18, 2014.

[11] X.-Y. Zhao, Q. Ao, L.-W. Yang, Y.-F. Yang, J.-C. Sun, and G.S. Gai, "Application of superfine pulverization technology in biomaterial industry," Journal of the Taiwan Institute of Chemical Engineers, vol. 40, no. 3, pp. 337-343, 2009.

[12] M. Li, J. H. Zhang, K. X. Zhu et al., "Effect of superfine green tea powder on the thermodynamic, rheological and fresh noodle making properties of wheat flour," LWT-Food Science and Technology, vol. 46, no. 1, pp. 23-28, 2012.
[13] X. Zhao, Z. Yang, G. Gai, and Y. Yang, "Effect of superfine grinding on properties of ginger powder," Journal of Food Engineering, vol. 91, no. 2, pp. 217-222, 2009.

[14] J. Hu, Y. Chen, and D. Ni, "Effect of superfine grinding on quality and antioxidant property of fine green tea powders," Food Science and Technology, vol. 45, no. 1, pp. 8-12, 2012.

[15] K. Takeda, M. Machida, A. Kohara, N. Omi, and T. Takemasa, "Effects of citrulline supplementation on fatigue and exercise performance in mice," Journal of Nutritional Science and Vitaminology, vol. 57, no. 3, pp. 246-250, 2011.

[16] C.-C. Huang, M.-C. Hsu, W.-C. Huang, H.-R. Yang, and C.C. Hou, "Triterpenoid-rich extract from antrodia camphorata improves physical fatigue and exercise performance in mice," Evidence-Based Complementary and Alternative Medicine, vol. 2012, Article ID 364741, 8 pages, 2012.

[17] J.-C. Chen, C.-Y. Hsiang, Y.-C. Lin, and T.-Y. Ho, "Deer antler extract improves fatigue effect through altering the expression of genes related to muscle strength in skeletal muscle of mice," Evidence-Based Complementary and Alternative Medicine, vol. 2014, Article ID 540580, 10 pages, 2014.

[18] M. Korivi, C. W. Hou, C. Y. Huang et al., "Ginsenoside-Rg1 protects the liver against exhaustive exercise-induced oxidative stress in rats," Evidence-Based Complementary and Alternative Medicine, vol. 2012, Article ID 932165, 8 pages, 2012.

[19] G. Lavanya, S. P. Voravuthikunchai, and N. H. Towatana, "Acetone extract from rhodomyrtus tomentosa: a potent natural antioxidant," Evidence-Based Complementary and Alternative Medicine, vol. 2012, Article ID 535479, 8 pages, 2012.

[20] S. Ataka, M. Tanaka, S. Nozaki et al., "Effects of Applephenon ${ }^{\circledR}$ and ascorbic acid on physical fatigue," Nutrition, vol. 23, no. 5, pp. 419-423, 2007.

[21] H. D. Choi, Y. K. Youn, and W. G. Shin, "Positive effects of astaxanthin on lipid profiles and oxidative stress in overweight subjects," Plant Foods for Human Nutrition, vol. 66, no. 4, pp. 363-369, 2011.

[22] F. Arredondo, C. Echeverry, J. A. Abin-Carriquiry et al., "After cellular internalization, quercetin causes Nrf2 nuclear translocation, increases glutathione levels, and prevents neuronal death against an oxidative insult," Free Radical Biology and Medicine, vol. 49, no. 5, pp. 738-747, 2010.

[23] J. Trujillo, Y. I. Chirino, E. Molina-Jijón, A. C. AndéricaRomero, E. Tapia, and J. Pedraza-Chaverrí, "Renoprotective effect of the antioxidant curcumin: recent findings," Redox Biology, vol. 1, no. 1, pp. 448-456, 2013. 


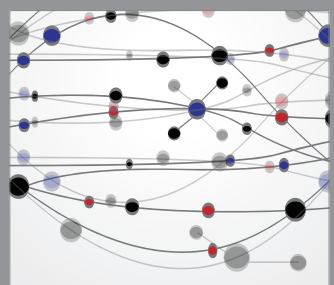

The Scientific World Journal
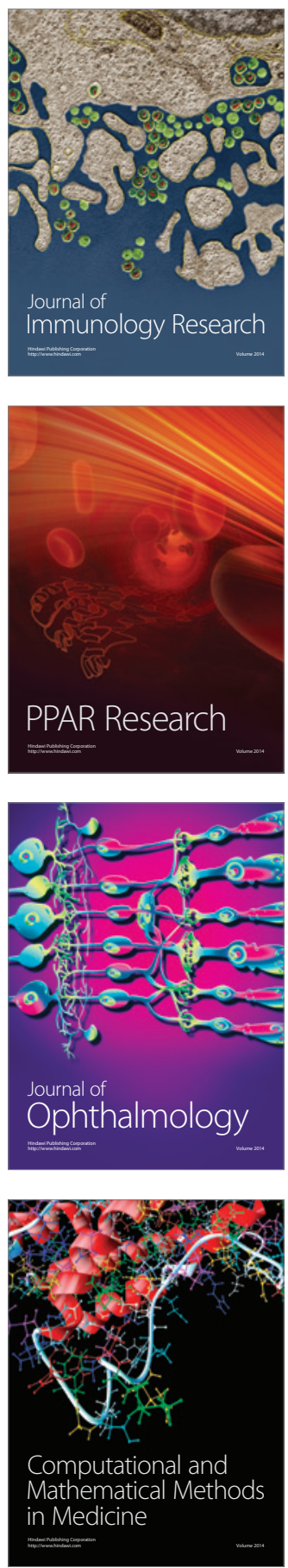

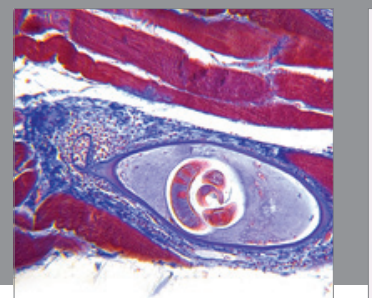

Gastroenterology

Research and Practice
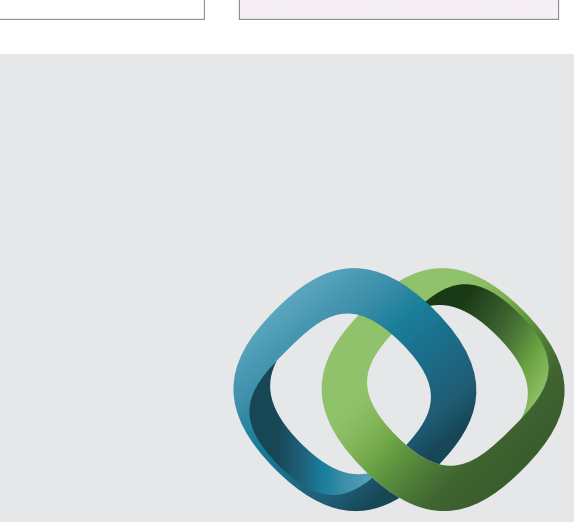

\section{Hindawi}

Submit your manuscripts at

http://www.hindawi.com
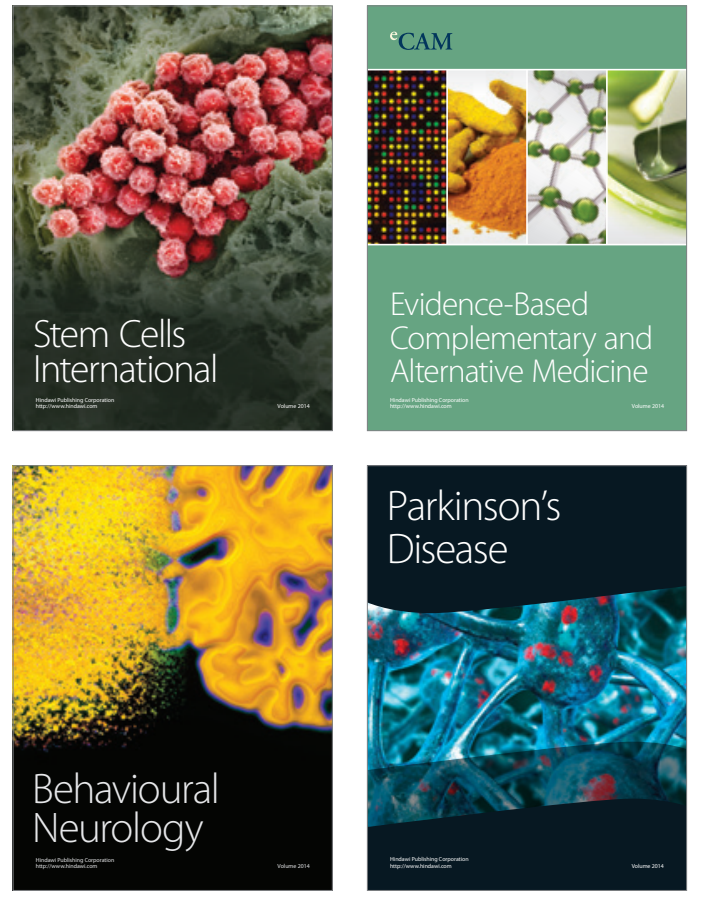
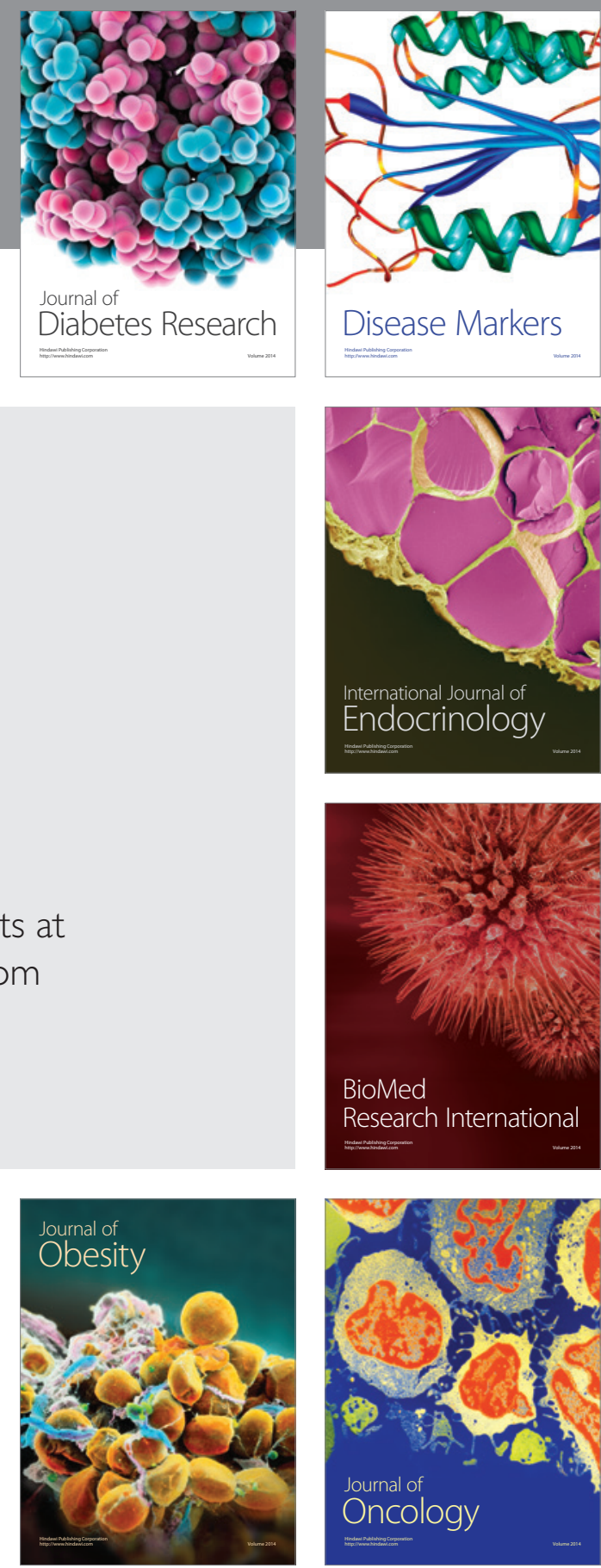

Disease Markers
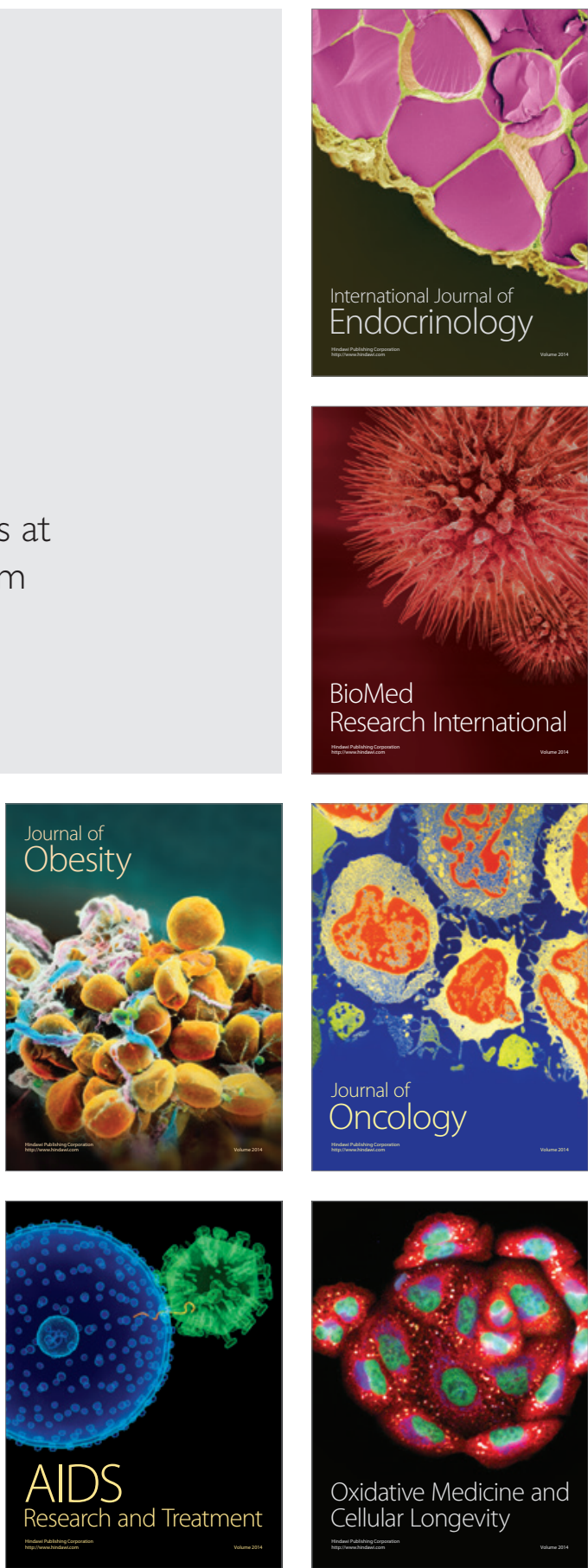